\title{
Informatics at UC Irvine
}

\author{
Paul Dourish \\ Department of Informatics \\ UC Irvine \\ Irvine, CA 92697-3440 USA \\ jpd@ics.uci.edu \\ Gillian R. Hayes \\ Department of Informatics \\ UC Irvine \\ Irvine, CA 92697-3440 USA \\ gillianrh@ics.uci.edu

\section{Lilly Irani} \\ Department of Informatics \\ UC Irvine \\ Irvine, CA 92697-3440 USA \\ lirani@ics.uci.edu

\section{Charlotte P. Lee} \\ Department of Informatics \\ UC Irvine \\ Irvine, CA 92697-3440 USA \\ cplee@ics.uci.edu \\ Silvia Lindtner \\ Department of Informatics \\ UC Irvine \\ Irvine, CA 92697-3440 USA \\ lindtner@ics.uci.edu \\ Bonnie Nardi \\ Department of Informatics \\ UC Irvine \\ Irvine, CA 92697-3440 USA \\ nardi@uci.edu

\section{Donald J. Patterson} \\ Department of Informatics \\ UC Irvine \\ Irvine, CA 92697-3440 USA \\ djp3@ics.uci.edu

\section{Bill Tomlinson} \\ Department of Informatics \\ UC Irvine \\ Irvine, CA 92697-3440 USA \\ wmt@ics.uci.edu \\ Copyright is held by the author/owner(s) \\ CHI 2008, April 5 - April 10, 2008, Florence, Italy \\ ACM 978-1-60558-012-8/08/04.
}

\begin{abstract}
Computer Science, as a single discipline, can no longer speak to the broad relevance of digital technologies in society. The Department of Informatics in the Donald Bren School of Information and Computer Sciences at the University of California, Irvine, serves as the institutional home for research on relationships between technological, organizational, and social aspects of information technology. Here, we describe the research landscape of the Department of Informatics and its relation to the diverse field of Human-Computer Interaction.
\end{abstract}

\section{Keywords}

Informatics, Research

\section{ACM Classification Keywords}

A.0 General; K4 Computers and Society; K.3.2

Computer and Information Science Education

\section{Introduction}

$\mathrm{HCI}$ as a field, and $\mathrm{CHI}$ as a conference, emerged in the 1980 s in response to changes in both information technologies and the contexts in which they were deployed. HCI arose at the intersection of cognitive psychology and computer science. It reflected a broadening of the relevance of computers and computer science. As information technologies have become increasingly central to everyday life, the scope 
The hallmark of our research is an empirical grounding in real-world practice.

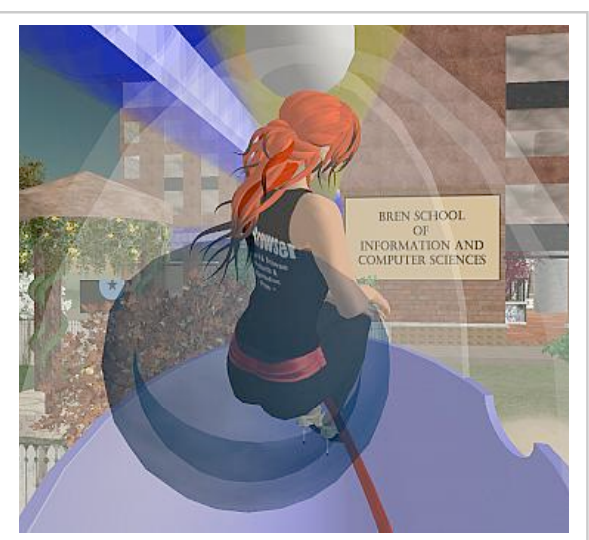

figure 1: Lopes's avatar travels in one of the virtual transporation "pods" she designed as part of the prototype system. of computer science and HCI interests has broadened. Informatics is the interdisciplinary study of the design, use, applications and implications of information and communication technology.

For several decades, UC Irvine's Department of Information and Computer Science had incorporated research on relationships between technological, organizational, and social aspects of information technology. More recently, the Department of Informatics has been formed to give these topics an institutional home. Our faculty members are specialists in computer science, psychology, anthropology, economics, arts, sociology, and more. The Bren School of ICS also houses the Departments of Computer Science and Statistics, so that the various approaches to studying information and information technology are brought together within a single institutional structure.

The fundamental focus of our research is a two-fold approach to information technology - as a means and as an object. As an object, information technology is something to be imagined, designed, created, and deployed; as a means, it is something that enables, constrains, amplifies, and transforms. Our interests lie in the relationship between these two aspects of interactive technology. Our program fuses a central focus on design with an abiding interest in the contexts within which designs are developed, deployed, and appropriated.

Our research, then, incorporates four connected programs: an empirical program of understanding technology design and use in practice; a theoretical program aimed at understanding contexts of information system use; a technological program aimed at new capabilities and infrastructures; and a design program focused on the integrative and holistic combination of technical and human considerations.

Past and present research projects present a multitude of examples of this approach. We conduct short and long-term fieldwork throughout the world, understanding the ways people adopt and integrate technology. We develop novel tools for specification of software architecture and study how they affect the social organization of software development projects in real organizations. We use empirical methods to understand people's constructions of privacy and security and support their practices and decisionmaking towards these goals. We create and use theoretical models of interaction that draw on social structures and organizational constraints coupled with technological possibilities and opportunities.

Through our engagements with public, private, governmental and non-profit organizations, our research connects to the world beyond the university. We have worked with venture capitalists, entrepreneurial think-tanks, and major open-source projects to spawn multiple ventures from our faculty and students. For example, an informatics alumnus, Roy Fielding, co-founded the Apache Server Project. Another alumnus, Jim Whitehead, headed the committee to develop the Distributed Authoring and Versioning (WebDAV) standard while he was still a doctoral student at UCI. As another example, Crista Lopes has been working with the Irvine-based transportation company Unimodal Inc. to create models of a commercially viable rapid-transit system, the SkyTran [6]. In addition to our expansive work in launching efforts, we work tightly with corporate 
research labs, such as Intel, IBM, Google, and Nokia. We also participate in rich collaborations with nonprofit organizations like AutismSpeaks and both non-profit and for-profit healthcare facilities, such as Children's Healthcare of Orange County.

\section{Background and History}

The Department of Informatics is currently housed within the Donald Bren School of Information and Computer Sciences. Founded as a department in 1968, Information and Computer Sciences (ICS) became a school in December 2002. It is home to the largest computing program in the UC system. Since its inception as a department, ICS has maintained an active research program in the application and impact of information technologies beyond the engineering concerns. The creation of the Department of Informatics in 2002 reinforces the central institutional role of innovative research that combines technological innovation with theoretically-informed social analysis.

In 2004, due to a generous gift from Donald Bren, the School was renamed in his honor. This gift included funding for ten endowed senior chairs, three targeted at interdisciplinary work. The new Bren School building, completed in 2006, includes 90,000 square feet of office, laboratory, and teaching space.

In addition to our specific research projects, faculty and students within the Department of Informatics and the Donald Bren School of ICS are involved in many programmatic activities that enhance the interdisciplinary and innovative atmosphere. Many of these activities cross-departmental, school, and university boundaries to create a unique research ecology.
For example, Informatics faculty helped found and continue to support the Arts Computation and Engineering (ACE) program, an interdisciplinary degree-granting program across the campus. The focus of the ACE curriculum is on criticality: historical, social, cultural and theoretical contextualization. Students and faculty engage in educational and research activities dedicated to real-time computational arts, interaction and interactivity.

As another example of Informatics faculty, researchers, staff and students using their core disciplinary strengths to cross institutional boundaries, many were integral to the founding and continued success of the California Institute for Telecommunications and Information Technology (Calit2). This institute extends the reach of the Internet throughout the physical world by teaming UC San Diego and UC Irvine faculty, students and research professionals with leading California telecommunications, computer, software and applications companies to conduct research on the scientific and technological components needed to bring this new Internet into being.

One of the latest initiatives to be spearheaded with the help of Informatics is the Center for Medical Informatics (CMI). Medical informatics research at UCI includes projects from across informatics, computer science, medicine, biology and public health. The focus includes pervasive and mobile computing for healthcare, computational biology, simulations, knowledge discovery, and probabilistic modeling and machine learning.

A huge number of other activities throughout campus are supported by the Informatics department at UCI- 


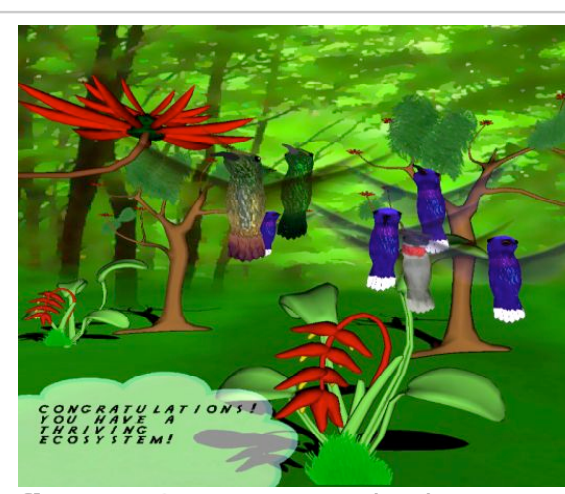

figure 2: Hummingbirds in a newly populated ecosystem as part of the EcoRaft project.

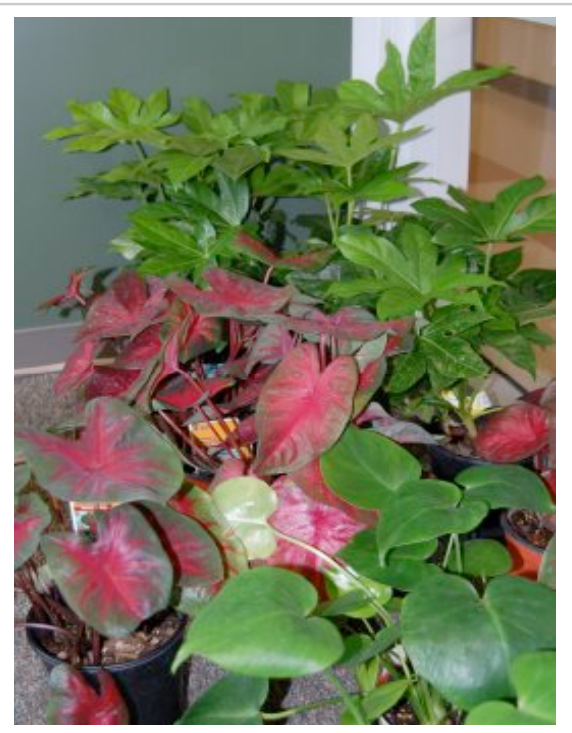

figure 3: Plants in the Technology Garden. too many to mention in a single document. What is significant about these activities, however, is that Informatics consistently serves as an organizational, professional, and academic interface between individuals and groups with different disciplinary strengths. We draw on the core of our own research and teaching, integrating concepts, methods, and findings from other areas.

\section{Empirical Grounding in Real-World Practices}

Research in the Department of Informatics covers a diverse range of topics. A consistent theme across many of these projects, regardless of the area, is our approach of using empirical grounding to study, understand, and support real-world practices. In this section, we introduce some of our recent projects.

\section{Merging Ecology with Technology}

Concepts surrounding sustainability and $\mathrm{CHI}$ have grown in importance as we as a community have begun to understand the impacts of our own technology development and the possibility of using technology to instruct and inform about sustainability.

The EcoRaft Project is an interactive educational exhibit designed to help people learn about restoration ecology through a hands-on interface. The project integrates stationary monitors and mobile tablet PCs that allow participants to move animated species between virtual islands easily and fluidly. EcoRaft demonstrates the principle that it is easy to destroy an ecosystem restoring it takes more work. With lifelike creatures, colorful visuals and a novel interface, EcoRaft showcases a merging of computing, arts, ecology, and education. A prototype of the project showed at SIGGRAPH 2005 in the Emerging Technologies program
[9], and the current prototype is slated to become a public exhibit at the Discovery Science Center in Orange County, CA.

In another effort, the Technology Garden, is a community maintained garden located in an office equipped with sensors. The Technology Garden supports dialog and thinking about how humans and plants relate to each other. By involving the Department broadly in the care and observation of a community garden located in an office, this project is exploring what role institutions may play in supporting sustainable activities and thinking. Through this work, the researchers wish to facilitate new forms of awareness and interaction among humans and nature through and with technology. The goals in creating the Technology Garden are threefold: (1) to promote thinking about ecological sustainability; (2) to create a pleasing office environment promoting relaxation; and (3) to encourage social interaction in an organization [4].

Medical and Health Informatics

Adoption, uses, and the potential for information technology in healthcare has long been a focus in the Department of Informatics. From studies of hospital workers [7] to understanding the needs of chronic cancer patients, clinicians, and caregivers [2], we continue to be involved in a wide range of medical and health informatics research.

A new project, UbiPlay, focuses on using Ubiquitous computing to facilitate activity and play. Through these methods, we hope to contribute to the growing research field dedicated to combating childhood obesity, the onset of diabetes, and more. We are working with the local school and the department of 


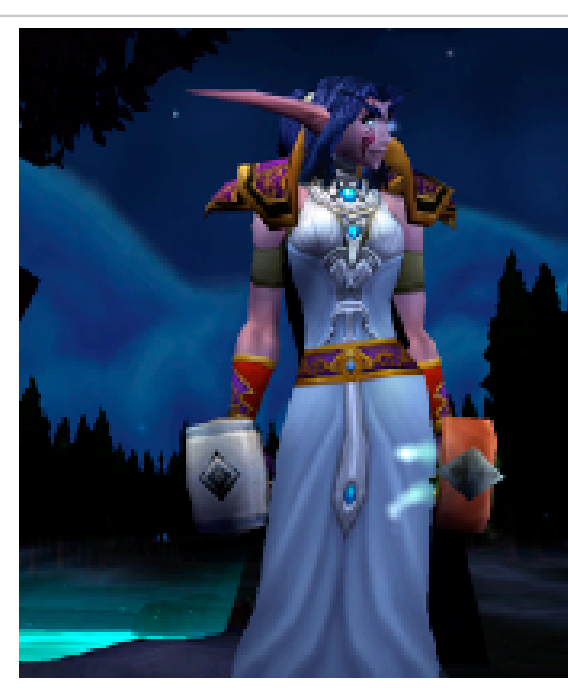

figure 4: Nardi's night elf priest, while conducting participant observation in World of Warcraft.
Pediatrics at UCI to understand how technology, media, and games contribute to choices about health. Working with games like Fish $\mathrm{N}^{\prime}$ Steps [5], physical video games like the Wii@ and Dance Dance Revolution $\odot$, and more this project will encourage not only physical activity but also increased understanding about nutrition and activity for children, adolescents, and adults.

Understanding and Supporting User Practices Surrounding Security and Privacy

Informatics researchers have long been at the forefront of work in privacy-sensitive design and understanding of practices surrounding the negotiation of privacy in a digital world. We have and continue to explore issues surrounding data access, control, surveillance, personalization and more from a variety of perspectives.

As one example of extensive research in security and privacy, Alfred Kobsa has directed an initiative for several years focus on privacy as a design requirement for personalized systems. This project studies specifically the impacts on "personalized" web-based systems, which cater their interaction to each individual user, collect considerable amounts of personal data for this purpose, and "lay them in stock" for possible future adaptation [3,12].

A collaboration between ICS and the schools of Social Ecology and Humanities at UCI under the direction of Paul Dourish has been conducting a study of paroled offenders tagged with GPS units as part of their parole conditions, providing an alternative perspective on the ways in which privacy and location-based systems are connected. Using a case study of paroled offenders tracked via Global Positioning System (GPS), researchers explored the ways that location-based technologies frame people's everyday experiences of space. In this work they introduce the notion of accountabilities of presence as an alternative to "privacy" that necessitates conceptualizations of mobility and privacy that are as complex, flexible and heterogeneous as social life itself [11].

\section{New Frontiers and Field Sites}

Informatics researchers are expanding field sites as HCI goes far beyond the traditional boundaries of offices and homes. We are actively working on research projects in online and developing worlds.

Our research extends beyond traditional online communities into massively-multiplayer online games (MMOG) and virtual worlds. For example, Bonnie Nardi's most recent research includes participantobservation fieldwork in World of Warcraft, the most popular MMOG, studying how players collaborate as well as the relationship of offline, online, and in-game activity [8]. Similarly, Ph.D. student Lilly Irani is studying an online disability activist group that uses a combination of virtual environments, social networking services, email, and blogs to stay connected. Meanwhile, Crista Lopes has been using the popular virtual world of Second Life as a prototyping platform, developing and testing new virtual transportation systems [6] and even conducting courses in the virtual world.

Informatics research also crosses international boundaries. For example, Prof. Don Patterson and students created Nomatic*Aid, a handheld geo-tagging photo device that enables coordination between human relief workers in Africa during, as well in the weeks and months after, crises. For an effective crisis response, spatial and temporal information about the resources 
(e.g. water, arable land, vaccines) and displaced people has to be collected and collated by the team of responders working on the field [1]. Meanwhile, Ph.D. student Amanda Williams is currently conducting fieldwork in Bangkok in order to examine topics in ubiquitous and urban computing from outside the traditional Western perspective.

\section{Summary and Future Directions}

Our premise is that information technology, as a part of everyday life, has moved beyond the traditional boundaries of "computer science." Informatics attempts to understand technology not simply in its own terms, but in terms of the social, organizational, and cultural settings in which it is produced, deployed, and used.

This is an inherently interdisciplinary endeavor, but universities are fundamentally organized along disciplinary lines. Rather than creating a program that crosses organizational boundaries, our approach has been to create a new institutional unit to house this work. Like the iSchools, we have sought to locate the interdisciplinary investigation of information technology within a common institutional infrastructure; but in contrast to most iSchools, we have attempted to do so in a structure that retains a strong link with technology design, both through our own design activities and through our institutional affiliation with Computer Science in a unified School.

\section{References}

[1] Aiyelokun, T., Patterson, D.J., et al. 2008. Understanding the Digital Divide in Southern Africa. Laboratory for Ubiquitous Computing and Interaction Tech Report LUCI-2008-002.
[2] Hayes, G.R., Abowd, G.D., et al. 2008. Opportunies for Pervasive Computing in Chronic Cancer Care. In Proc. of Pervasive 2008. Sydney, Australia, May 2008.

[3] Kobsa, A. 2007. Privacy-enhanced Personalization. Communications of the ACM (50).

[4] Lee, C.P., Hornick, B., et al. 2008. The Technology Garden. Laboratory for Ubiquitous Computing and Interaction Tech Report LUCI-2008-001. 2008.

[5] Lin, J., Mamykina, L., et al., 2006. Fish'n'Steps: Encouraging Physical Activity with an Interactive Computer Game, in Proc of Ubicomp '06. (Newport Beach, California, USA).

[6] Lopes, C., Kan, L., et al. 2008. PRT Simulation in an Immersive Virtual World, in Proc. of SIMUTools'08, Marseille, France, March 2008.

[7] Reddy, M. and Dourish, P. 2002. A Finger on the Pulse: Temporal Rhythms and Information Seeking in Medical Work. Proc. of CSCW 2002 (New Orleans, LA), 344-353. New York: ACM.

[8] Nardi, B. A., Ly, S., and Harris, J. 2007. Learning Conversations in World of Warcraft. Proc. of HICSS 2007. IEEE Computer Society, Washington, DC, 79.

[9] Tomlinson, B.M. L. Yau and J. Gray. 2005. Heterogeneous Character Animation: How to make an interactive character jump between stationary and mobile graphical computers In: SIGGRAPH 05 Sketches.

[10] Tomlinson, B., Gray, J., et al. 2005. "Multiple Virtual Rafts: A Multi-User Paradigm for Interacting with Communities of Autonomous Characters." In: ACM Conference On Human Factors In Computing Systems (CHI 2005), Late Breaking Results, Portland, OR.

[11] Troshynski, E., Lee, C., and Dourish, P. 2008. Accountabilities of Presence: Reframing Location-Based Systems. In Proc of CHI 2008 (Florence, Italy).

[12] Wang, Y. and Kobsa, A. 2007. Respecting Users' Individual Privacy Constraints in Web Personalization. Proc. of User Modeling 2007: 157-166. 